\title{
Power Quality Disturbances Detection and Classification Rule-Based Decision Tree
}

\author{
FOUAD R. ZARO \\ Electrical Engineering Department, \\ Palestine Polytechnic University, \\ Hebron, PALESTINE
}

\begin{abstract}
In this paper, the power quality (PQ) disturbances have been detected and classified using Stockwell's transform (S-transform) and rule-based decision tree (DT) according to IEEE standards. The proposed technique based on the extracted features of the PQ events signals, which are extracted from the timefrequency analysis. Several PQ disturbances are considered with simple and complex disturbances to include spike, flicker, oscillatory transient, impulsive transient, and notch. The performance and robustness of the proposed technique for the recognition of PQ disturbances have been demonstrated through the results of the various disturbances. By comparing the performance of the proposed technique with other reported studies it was distinguished results under noiseless and noisy conditions
\end{abstract}

Keywords - stockwell Transform, Feature Extraction, Time Frequency Analysis, Matlab Program, Power Quality Disturbance, Rule-based Decision Tree.

Received: November 5, 2020. Revised: March 5, 2021. Accepted: March 27, 2021. Published: April 1, 2021.

\section{Introduction}

Increasing use of Equipment and appliances that nonlinear, computers, variable speed drives and solid-state switching devices in the electrical network and the multiplicity and different types of consumers like residential, commercial, industrial and agricultural have been led to the important issue of power quality each both customers and utilities [1].

It all causes power quality disturbances events, that leads to distortion of voltage and current signals because of the effect PQ disturbances such as harmonics, flicker, sag, swell, notches, transient, interruption, spike, impulsive transient, unbalance voltage and current [2]. The effect of poor in PQ is miss operation of equipment and devices, failures of motors, overheating in lines and equipment, interruption in power supply on devices, or destroy this device and decreasing efficiency [3].

It's so important to know the type of PQ disturbances before PQ improvement action, through detection and classification PQ disturbances and localized it.

Many of intelligent artificial and mathematical advanced process signals are proposed to classification and to detection PQ disturbances that which features overlap each other [4]. some of these techniques used to classify PQ disturbance are Fourier transforms (FT), short-time Fourier transform (STFT), wavelet transform (WT), continuous wavelet transforms (CWT), and discrete wavelet transforms (DWT), but WT is more efficient and successful than FT and STFT, where found the STFT is not much success to classify PQ disturbance.

etc.
However, CWT and DWT more efficient to analysis transient, but theses techniques are not efficient to analysis PQ disturbance signal with electrical noise [5][7]. The WT dyadic-orthonormal to classify and localize PQ disturbances has been proposed in [8].

Stockwell's transform combine between features of both WT and STFT, it depends on the window that width decrease with the frequency and provides resolution depending on frequency [9], ST provides a high accuracy even in the high level of noise [10]-[11]. ST can be used for online monitoring of PQ disturbance in the network to detect and recognition disturbances [12].

Support vector machine (SVM) and artificial neural network (ANN) have been used for automatic classification of PQ disturbances based on ST [13]-[15].

In 1980 invented the detection tree algorithm by Breiman and this algorithm has considered as classifier tool supported by the decision rules. the first scientist implement DT is Wehenkel in 1989 and used this algorithm in the power system filed [16]. the DT presented as a binary graph tree that used to detection unknown relationships between input and output parameters [17]. DT drown graph like tree starting from the root node down to a leaf node. the response and result of DT there are in the leaf node and root node has the first edition rules [18].

In this paper a method of extraction feature for PQ disturbances detection and classification from time 
frequency representation using ST has been presented. The proposed classifier use of these features that rulebased DT to classify various PQ disturbances. As well as, the performance of the proposed algorithm has been studied with noise and efficiency comparison between the proposed method and other methods used in the literature has been carried out.

\section{Stockwell Transform}

In 1994 Stockwell proposed transform signal processing name it Stockwell transform, the Stockwell transform contained a mix of elements of both WT and STFS but ST located at different categories [19].

A signal varying with time like PQ disturbances signal can be analyzed by ST by using MAR while kept the absolute phase of each frequency [20]-[21]. So, ST used effectively to analyze signal that varies with time like PQ disturbances. The ST is defined as:

$$
\begin{gathered}
S(\tau, f)=\int_{-\infty}^{\infty} h(t) w(\tau-t, f) e^{-i 2 \pi f t} d t \\
w(t, f)=\frac{|f|}{\sqrt{2 \pi}} e^{-t^{2} f^{2} / 2}
\end{gathered}
$$

Where

$w(t, f)$ is the Gaussian window function

$\sigma(f)=\frac{1}{|f|}$ is the window width.

S-matrix is a complex matrix represents the output of S-transform with size $\mathrm{n} \times \mathrm{m}$. S-matrix (Rows): represents the time-domain distribution of the signal in the particular frequency. S-Matrix (columns): represents the amplitude frequency characteristics.

the S-matrix makes us able to extract important information of the signal in some certain time in terms of magnitude, frequency and phase, as well as extract many important curves for the signal like: curves of amplitude, phase, the sum of absolute values, amplitude-frequency and time-frequency contour.

\section{Proposed methodology}

Rule-based decision tree algorithm will be used for detection, classification and localization of the PQ disturbances as shown in Fig. 1, below.

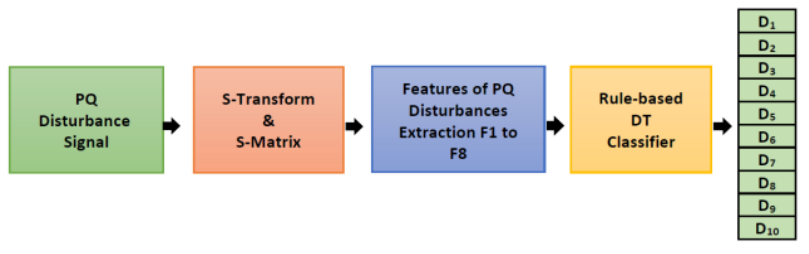

Figure 1. Blok diagram of PQ disturbance by using rule-based decision tree.
The Mathematical models of PQ disturbances signals has been obtained from the IEEE-1159 standard [22], and then have been generated by Matlab after that analysis of these signals by multi-resolution analysis (MRA) based on S-transform to get a complex S-matrix. all features $F_{1}$ to $F_{9}$ extraction from $S$-matrix, where $F_{1}$ to $F_{8}$ is used to obtain the rules of the decision tree, whereas $\mathrm{F}_{9}$ presented the localize the PQ disturbances.

\section{PQ Disturbance Analysis Using S-Transform}

In this part the analysis of various PQ disturbances based on the features extracted from ST will be presented. The disturbance signals have been generated based on IEEE-1159 standard using MATLAB program. Table I, below represents the mathematical modeling for the generated PQ signals:

TABLE I. MATHEMATICAL MODEL OF PQ DistURBANCES.

\begin{tabular}{|c|c|c|c|}
\hline $\mathrm{S}$ & Type & Equation & Parameters \\
\hline $\mathrm{S}_{1}$ & $\begin{array}{c}\text { Pure sine } \\
\text { wave }\end{array}$ & $\sin (\omega \mathrm{t})$ & $f=50 H z, \omega=2 \pi \mathrm{f}$ \\
\hline $\mathrm{S}_{2}$ & Flicker & $\begin{array}{c}\mathrm{h}(\mathrm{t})=(1+\alpha \mathrm{f} \\
\sin (\beta \omega \mathrm{t})) \sin (\omega \mathrm{t})\end{array}$ & $0.1 \leq \alpha \mathrm{f} \leq 0.2,5 \mathrm{~Hz} \leq \beta \leq 20 \mathrm{~Hz}$ \\
\hline $\mathrm{S}_{3}$ & $\begin{array}{c}\text { Oscillator } \\
\mathrm{y} \text { transient }\end{array}$ & $\begin{array}{c}\mathrm{h}(\mathrm{t})=\sin (\omega \mathrm{t})+ \\
\alpha \sin (\omega \mathrm{t}-\mathrm{t} 1) / \tau \\
(\mathrm{t}-\mathrm{t} 1)(\mathrm{u}(\mathrm{t} 2)-\mathrm{u}(\mathrm{t} 1)\end{array}$ & $\begin{array}{c}0.1 \leq \alpha \leq 0.8,0.5 \mathrm{~T} \leq \mathrm{t} 2-\mathrm{t} 1 \\
\leq 3 \mathrm{~T}, \tau=(\mathrm{t} 1+\mathrm{t} 2) / 2,300 \mathrm{~Hz} \leq \\
\mathrm{fn} \leq 900 \mathrm{~Hz}\end{array}$ \\
\hline $\mathrm{S}_{4}$ & $\begin{array}{c}\text { Impulsive } \\
\text { transient }\end{array}$ & $\begin{array}{c}\mathrm{h}(\mathrm{t})=\sin (\omega \mathrm{t}) \\
\operatorname{sgn}[\sin (\omega \mathrm{t})]^{*} \alpha( \\
\mathrm{u}(\mathrm{t} 2)-\mathrm{u}(\mathrm{t} 1))\end{array}$ & $\begin{array}{c}0.1 \leq \alpha \leq 0.4,0.05 \mathrm{~T} \leq \mathrm{t} 2-\mathrm{t} 1 \\
\leq 0.2 \mathrm{~T}\end{array}$ \\
\hline $\mathrm{S}_{5}$ & Notch & $\begin{array}{c}\mathrm{h}(\mathrm{t})=\sin (\omega \mathrm{t})- \\
\mathrm{sgn}[\sin (\omega \mathrm{t})]^{*} \alpha( \\
\mathrm{u}(\mathrm{t} 2)-\mathrm{u}(\mathrm{t} 1))\end{array}$ & $\begin{array}{c}0.1 \leq \alpha \leq 0.4,0.05 \mathrm{~T} \leq \mathrm{t} 2-\mathrm{t} 1 \\
\leq 0.2 \mathrm{~T}\end{array}$ \\
\hline $\mathrm{S}_{6}$ & Spike & $\begin{array}{c}\mathrm{h}(\mathrm{t})=\sin (\omega \mathrm{t}) \\
\operatorname{sgn}[\sin (\omega \mathrm{t})]^{*} \alpha( \\
\mathrm{u}(\mathrm{t} 2)-\mathrm{u}(\mathrm{t} 1))\end{array}$ & $\begin{array}{c}0.1 \leq \alpha \leq 0.4,0.005 \mathrm{~T} \leq \mathrm{t} 2-\mathrm{t} 1 \\
\leq 0.02 \mathrm{~T}\end{array}$ \\
\hline
\end{tabular}

All the generated signals with frequency of $50 \mathrm{~Hz}, 10$ cycles and sampling frequency of $3.2 \mathrm{kHz}$. The disturbance signals was denoted by symbols $\mathrm{S}_{2}$ to $\mathrm{S}_{12}$, while the pure sine wave was denoted by $S_{1}$, these signals was analyzed by S-matrix.

\subsection{Pure sine Wave}

The plots obtained for the pure sine wave is considered as a reference for PQ disturbance detection. All curves were of constant amplitude except for amplitudefrequency curve which showed one amplitude appeared at $50 \mathrm{~Hz}$.

\subsection{Flicker}

Voltage flicker and related ST plots are shown in Fig. 5. The voltage flicker can be recognized from a series of circles in the frequency contour as shown in Fig. 2, (b) and a continuous ripple the sum of absolute values curve 
Fig. 2, (d) also a finite value between 0.25 to 0.4 of the normalized frequency predicts the presence of flicker.

Figure 2 shows plots of the flicker signal and ST related, the series circles showed in the S-contour curve, and continuous replies in sum absolute values curve their indicators to detect flicker, In Figure 2 (f) the amplitude-

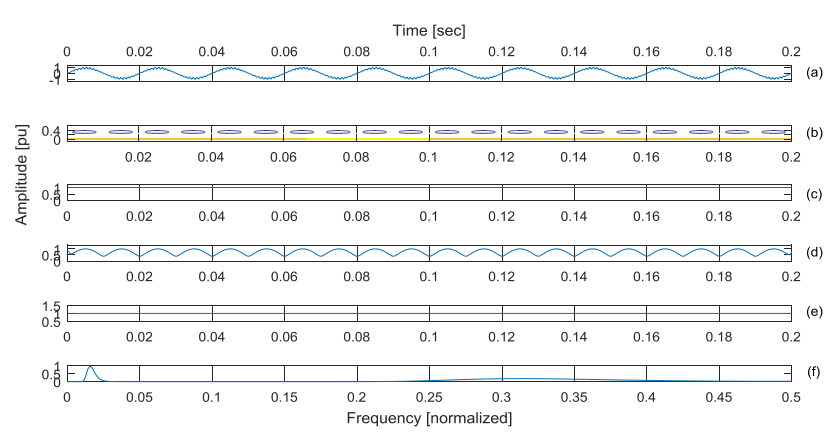

frequency curve has two peaks. a finite value frequency between 0.25 to 0.40 of the normalized frequency predicts the presence of flicker.

Figure 2. (a) Flicker (b) frequency contour (c) amplitude-time curve (d) sum absolute values curve (e) phase curve (f) amplitude-frequency curve.

\subsection{Oscillatory Transient}

Figure 3 shows the S-transform plots of oscillatory transient event. As shown in Figure 3(b) by single isolated contour in the frequency contour oscillatory transient can be depicted, also changes in all curves can indicate the presence of this disturbance. Localization is achieved by the second derivative of the sum of absolute values curve Figure 3(e).

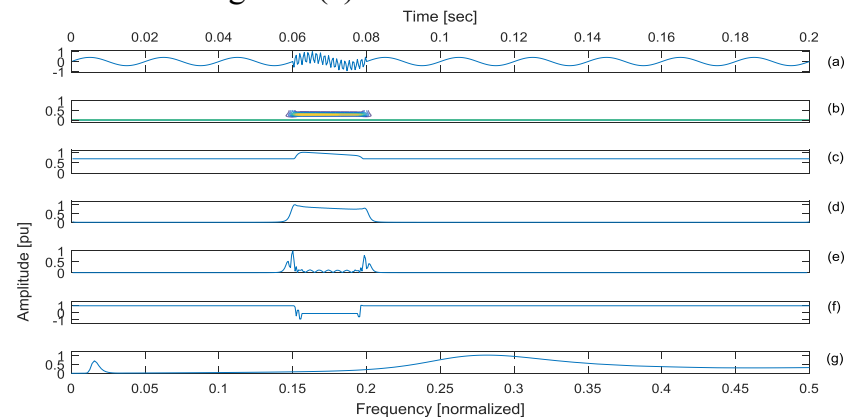

Figure 3. (a) Oscillatory transient (b) frequency contour (c) amplitudetime curve (d) sum absolute values curve (e) second derivative of sum absolute values curve (f) phase curve (g) amplitude-frequency curve.

\subsection{Impulsive Transient}

Figure 4 shows impulsive transient and related plots according to S-transform. Impulsive transient event in any signal can be depicted from frequency contour plot as shown in Figure 4(b) as well as from sum of absolute values curve as shown in Figure 4(d).

The impulsive transient event usually contains all the frequency components thus a considered value of frequency within the accepted range of frequency is noticed in the amplitude frequency curve as shown in Figure 4(g).

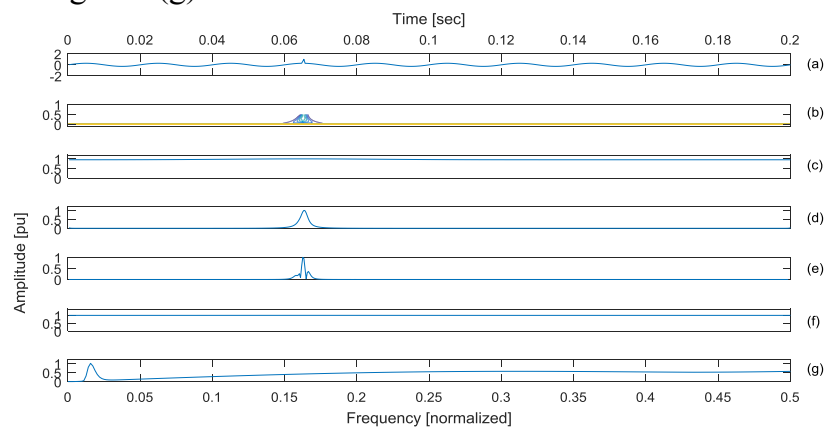

Figure 4. (a) Impulsive transient (b) frequency contour (c) amplitudetime curve (d) sum absolute values curve (e) second derivative of sum absolute values curve (f) phase curve (g) amplitude-frequency curve.

\subsection{Multiple Notches}

According to S-transform the plots of the multiple notches event are shown in Figure 5. This event can be detected by the chain of discontinuous contours in the frequency-contour as shown in Figure 5(b) and by series of repeated peaks as illustrated in Figure 5(d) which represents the sum of the absolute values curve. Moreover, the ascending increase in the amplitude of frequency through all the frequency range as shown in Figure $5(\mathrm{~g})$ which depicts the amplitude frequency-curve is an indication for the presence of notches.

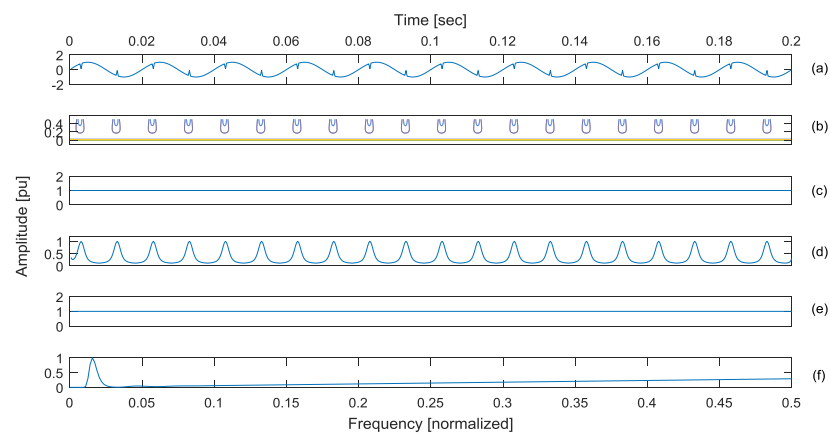

Figure 5. (a) Multiple Notches (b) frequency contour (c) amplitude-time curve (d) sum absolute values curve (e) phase curve (f) amplitudefrequency curve.

\subsection{Multiple Spikes}

Figure 6 shows the plots of multiple spikes event depending on S-transform results. All the plots are the same as in the notches. However, the number of contours in Figure 6(b) and the number of peaks in Figure 6(d) rely on the number of spikes in the signal. 


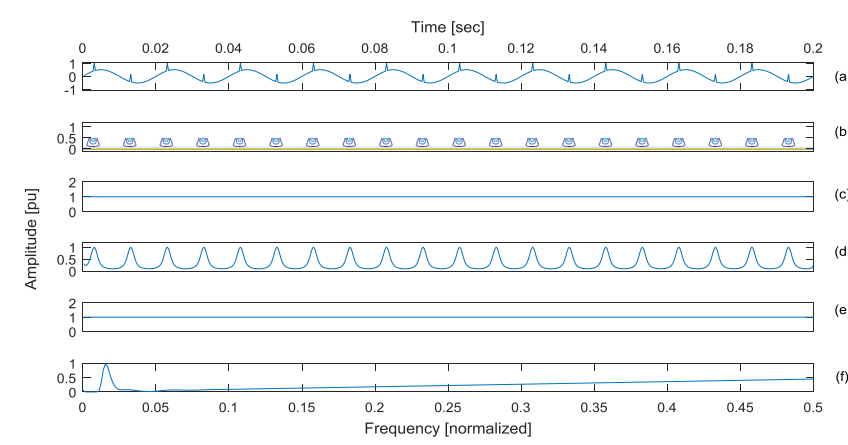

Figure 6. (a) Multiple Spikes (b) frequency contour (c) amplitude-time curve (d) sum absolute values curve (e) phase curve (f) amplitudefrequency curve.

\section{Feature Extraction}

The proposed approach for detection of power quality events is based on the extracted feature from S-transform results. In this study, these features are labelled as $F_{1}$, $\mathrm{F}_{2} \ldots \mathrm{F}_{9}$. The extracted features can be defined as follows: $\mathrm{F}_{1}$ : Sum factor $\left(S_{f}\right)$

$S_{f}=\max (S)+\min (S)-\max (R)-\min (R)$

Where $S$ represents the data array collected from the sum of the absolute values of the distorted signal. $R$ represents the data array collected from the sum of the absolute values of the pure sinusoidal signal.

$F_{2}$ : Number of peaks in the frequency amplitude curve. $\mathrm{F}_{3}$ : Skewness of phase curve. Skewness of a signal is defined as:

$$
S=\frac{E(x-\mu)^{3}}{\sigma^{3}}
$$

Where $x$ refers to the data array of signal, $\mu$ : mean of $x$, $\sigma$ : standard deviation of $x$, and $E$ : expected value of the quantity.

$\mathrm{F}_{4}$ : Amplitude factor $A_{f}=(1+(C-A)+(D-B))$ where $C$ is the maximum value of the amplitude curve of the distorted signal. $D$ is the minimum value of amplitude curve of the distorted signal. $A$ is the maximum value of the amplitude curve of the pure sinusoidal signal. $B$ is the minimum value of amplitude curve of the pure sinusoidal signal.

$\mathrm{F}_{5}$ : Kurtosis of amplitude curve. The Kurtosis of a signal is given by the following formula:

$$
k=\frac{E(x-\mu)^{4}}{\sigma^{4}}
$$

Where $\mathrm{x}$ : array of data of signal, $\mu$ : mean of $\mathrm{x}, \sigma$ : standard deviation of $\mathrm{x}$, and $\mathrm{E}$ : expected value of the quantity.
$\mathrm{F}_{6}$ : Kurtosis of phase curve.

$\mathrm{F}_{7}$ : Kurtosis of sum absolute values curve.

$\mathrm{F}_{8}$ : Kurtosis of amplitude-frequency curve.

$\mathrm{F}_{9}$ : Second order derivative of sum absolute values curve:

$$
F 9=\frac{\partial^{2} f}{\partial n^{2}}=f(n+1)+f(n-1)-2 f(n)
$$

Where $f(n)$ represents the sum of the absolute values of the signal. $n$ refers to the sample number. The power quality events can be localized according to the detected spikes using the feature $\mathrm{F}_{9}$.

\section{Detection and classification using rule-based decision tree}

The adopted classification technique in this study for power quality disturbances is built on the rule-based decision tree (RBDT) and depending on the extracted features $F_{1}$ to $F_{8}$ from the S-transform.

When the value of feature $F_{1}$ equals zero that indicates no distortion in the tested signal. On the other hand, when the value of feature $F_{1}$ equals a finite value indicates a distortion in the tested signal.

Table II contains the numerical values of the extracted features $\left(\mathrm{F}_{1}\right.$ to $\left.\mathrm{F}_{8}\right)$ depending on the S-transform results. These values have been used in building the RBDT.

According to the extracted feature $\left(\mathrm{F}_{2}\right)$ that represents the number of peaks in the amplitude-frequency curve the first signals are classified into two main groups. In the first group, the value of $F_{2}$ equals 1 , whereas in the second group, it's more than 1. Under each group there are several subgroups based on the value of $F_{1}$. The classification process of PQ events continues till the end of the rules. The flowchart of the proposed algorithm is illustrated in Figure 7.

The threshold values have been selected depending on the MRA of the S-transform. The performance and efficiency of the proposed technique is summarized

\begin{tabular}{|c|c|c|c|c|c|}
\hline \multirow[b]{2}{*}{ PQ event } & \multirow{2}{*}{$\begin{array}{l}\stackrel{\Xi}{\Xi} \\
\stackrel{\Xi}{0}\end{array}$} & \multicolumn{2}{|c|}{$\begin{array}{c}\text { Correctly } \\
\text { Classified } \\
\end{array}$} & \multicolumn{2}{|c|}{ Efficiency (\%) } \\
\hline & & $\begin{array}{l}\text { Without } \\
\text { noise }\end{array}$ & $\begin{array}{c}20 \\
\mathrm{~dB} \\
\text { SNR }\end{array}$ & $\begin{array}{l}\text { Without } \\
\text { noise }\end{array}$ & $\begin{array}{c}20 \mathrm{~dB} \\
\text { SNR }\end{array}$ \\
\hline Pure sine wave & $\mathrm{S}_{1}$ & 100 & 100 & $100 \%$ & $100 \%$ \\
\hline Flicker & $\mathrm{S}_{2}$ & 98 & 98 & $98 \%$ & $98 \%$ \\
\hline $\begin{array}{l}\text { Oscillatory } \\
\text { transient }\end{array}$ & $\mathrm{S}_{3}$ & 99 & 99 & $99 \%$ & $99 \%$ \\
\hline $\begin{array}{l}\text { Impulsive } \\
\text { transient }\end{array}$ & $\mathrm{S}_{4}$ & 100 & 97 & $100 \%$ & $97 \%$ \\
\hline Notch & $\mathrm{S}_{5}$ & 100 & 100 & $100 \%$ & $100 \%$ \\
\hline Spike & $\mathrm{S}_{6}$ & 98 & 96 & $98 \%$ & $96 \%$ \\
\hline \multicolumn{4}{|c|}{ Overall Efficiency } & $99.3 \%$ & $98.1 \%$ \\
\hline
\end{tabular}
clearly in Table III.

TABLE III. RULE-BASED DeCision TREe ClaSSIFICATION RESUlT 


\section{Performance comparison}

The performance of the proposed technique has been compared with others techniques proposed in references as tabulated in Table IV. Evidently, the higher performance is for the proposed algorithm.
TABLE IV. PERFoRMANCE COMPARISON

\begin{tabular}{|c|c|c|c|c|}
\hline \multirow{2}{*}{ Reference } & \multirow{2}{*}{$\begin{array}{c}\text { Type of } \\
\text { algorithm }\end{array}$} & \multirow{2}{*}{$\begin{array}{c}\text { No. of } \\
\text { Compared } \\
\text { PQ }\end{array}$} & \multicolumn{2}{|c|}{$\begin{array}{c}\text { Overall } \\
\text { Efficiency (\%) }\end{array}$} \\
\cline { 4 - 5 } & & disturbances & $\begin{array}{c}\text { without } \\
\text { noise }\end{array}$ & $\begin{array}{c}20 \mathrm{~dB} \\
\text { SNR }\end{array}$ \\
\hline$[6]$ & $(\mathrm{ST}+\mathrm{TT}+\mathrm{ANN})$ & $\mathrm{S} 3$ & $92.1 \%$ & - \\
\hline$[14]$ & $(\mathrm{SSD}+\mathrm{HD})$ & $\mathrm{S} 5$ & $96.7 \%$ & $95.4 \%$ \\
\hline Proposed & $(\mathrm{ST}+\mathrm{DT})$ & & 99.14 & 98.20 \\
\hline
\end{tabular}

TABLE II. S- TRANSFORM BASED FEATURES OF PQ DistURBANCES

\begin{tabular}{|c|c|c|c|c|c|c|c|c|c|}
\hline \multirow{2}{*}{ PQ Disturbance } & \multirow{2}{*}{ PQ Symbol } & \multicolumn{8}{|c|}{ Features of PQ Disturbances } \\
\hline & & $\mathrm{F}_{1}$ & $\mathrm{~F}_{2}$ & $\mathrm{~F}_{3}$ & $\mathrm{~F}_{4}$ & $\mathrm{~F}_{5}$ & $\mathrm{~F}_{6}$ & $\mathrm{~F}_{7}$ & $\mathrm{~F}_{8}$ \\
\hline Pure sine wave & $\mathrm{S}_{1}$ & 0 & 1 & -1.3514 & 1 & 2.0317 & 1.9206 & 242.034 & 74.03 \\
\hline Flicker & $\mathrm{S}_{2}$ & 4.827 & 2 & 7.8275 & 1 & 2.1881 & 4.4676 & 1.739 & 33.7382 \\
\hline Oscillatory transient & $\mathrm{S}_{3}$ & 30.3679 & 2 & 1.4619 & 1.3123 & 11.5043 & 11.427 & 7.6898 & 2.4878 \\
\hline Impulsive transient & $\mathrm{S}_{4}$ & 36.5376 & 3 & 1.0537 & 1.1139 & 2.6907 & 2.5511 & 43.6471 & 3.3385 \\
\hline Notch & $\mathrm{S}_{5}$ & 10.6233 & 1 & -0.9528 & 0.9248 & 2.4089 & 2.4088 & 3.0134 & 19.3115 \\
\hline Spike & $\mathrm{S}_{6}$ & 15.489 & 1 & 1.094 & 1.0159 & 2.6476 & 2.6584 & 8.3565 & 7.7361 \\
\hline
\end{tabular}

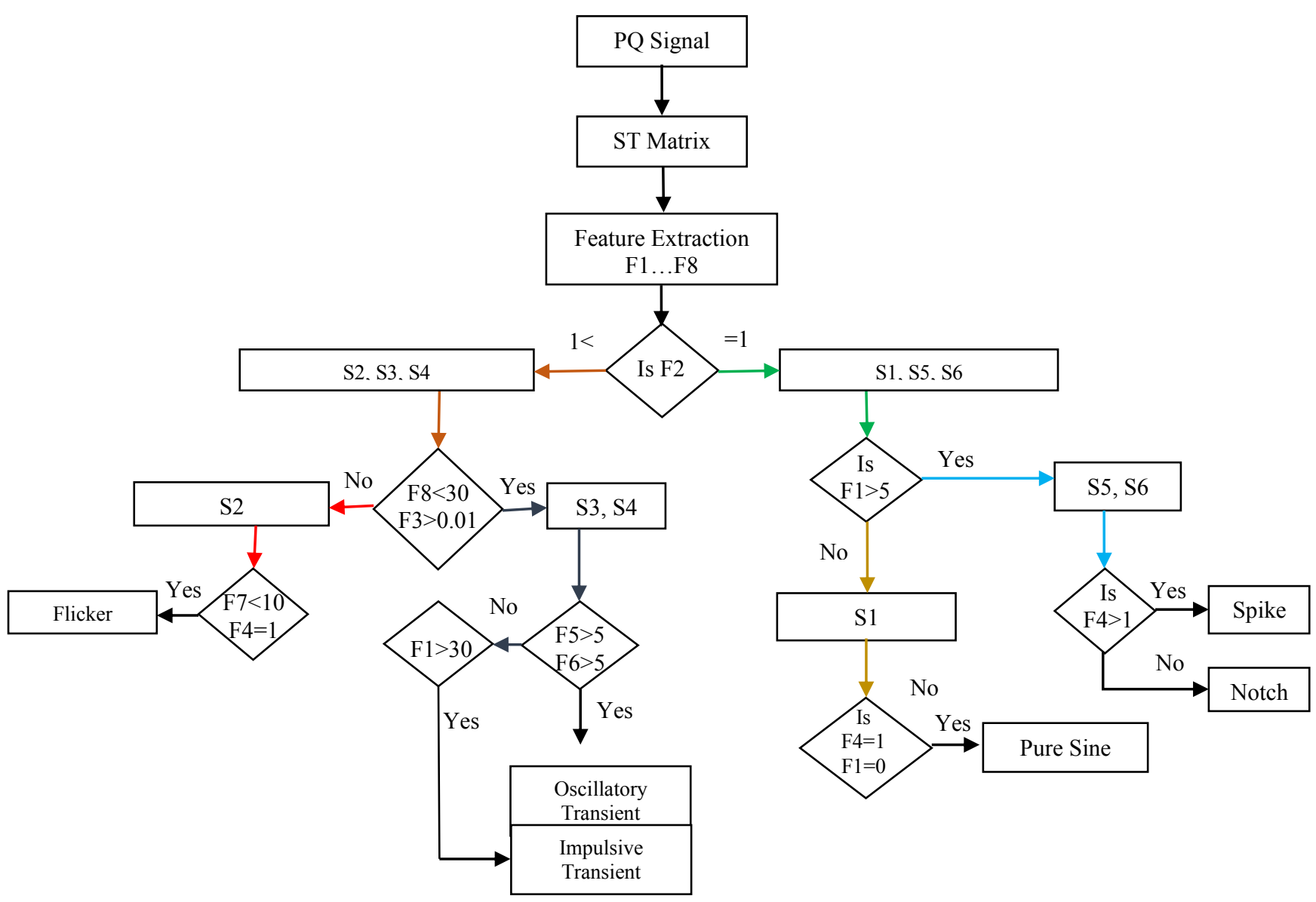

Figure 7: Block diagram for classification of power quality disturbance.

8. CONCLUSION
The proposed technique for detection and classification of the power quality disturbances has been effectively done based on the extracted features from the S-transform 
labeled $F_{1}$ to $F_{8}$ to create the rule-based decision tree. Whereas, the extracted feature $\mathrm{F}_{9}$ has been used for the localization of power quality events in time. The superiority and effectiveness of the proposed technique have been tested over hundreds of data sets for each PQ event as well as the results proved to have an efficiency greater than $98 \%$ even in a noisy environment.

\section{References}

[1] M. Uyar, S. Yildirim, and M. T. Gencoglu, "An expert system based on s-transform and neural network for automatic classification of power quality disturbances," Expert Systems with Applications, vol. 36, no. 3, Part 2, pp. $5962-5975,2009$.

[2] B. Biswal, H. Behera, R. Bisoi, and P. Dash, "Classification of power quality data using decision tree and chemotactic differential evolution based fuzzy clustering," Swarm and Evolutionary Computation, vol. 4, pp. $12-24,2012$.

[3] M. Masoum, S. Jamali, and N. Ghaffarzadeh, "Detection and classification of power quality disturbances using discrete wavelet transform and wavelet networks," Science, Measurement Technology, IET, vol. 4, no. 4, pp. 193-205, July 2010.

[4] O. P. Mahela, A. G. Shaik, and N. Gupta, "A critical review of detection and classification of power quality events," Renewable and Sustainable Energy Reviews, vol. 41, pp. 495 - 505, 2015. [Online]. Available: http://www.sciencedirect.com/science/article/pii/S1364032114007 $\underline{564}$

[5] O. P. Mahela, A. G. Shaik, N. Gupta, A critical review of detection and classi_cation of power quality events, Renewable and Sustainable Energy Reviews 41 (2015) 495 \{ 505. doi:http://dx.doi.org/10.1016/j.rser. 2014.08.070.

[6] D. Granados-Lieberman, R. J. Romero-Troncoso, R. A. Osornio-Rios, Garcia-Perez, E. Cabal-Yepez, Techniques and methodologies for power quality analysis and disturbances classification in power systems: a review, IET Generation, Transmission Distribution 5 (4) (2011) 519\{529. doi:10.1049/iet-gtd.2010.0466.

[7] P. Thakur, A. K. Singh, Signal processing and ai based diagnosis of power quality disturbances: A review, in: Energy Economics and Environment (ICEEE), 2015 International Conference on, 2015, pp. $1\{6$. doi:10.1109/ EnergyEconomics.2015.7235071.

[8] S. Santoso, E. Powers, W. Grady, and P. Hofmann, "Power quality assessment via wavelet transform analysis," Power Delivery, IEEE Transactions on, vol. 11, no. 2, pp. 924930, Apr 1996.

[9] Singh, D. Shahani, A. Chandra, and K. Al-Haddad, "Recognition of power-quality disturbances using stransform-based ANN classifier and rule-based decision tree," Industry Applications, IEEE Transactions on, vol. 51, no. 2, pp. 1249-1258, March 2015

[10] R. Kumar, B. Singh, D. T. Shahani, A. Chandra, K. AlHaddad, Recognition of power-quality disturbances using s-transform-based ANN classifier and rule-based decision tree, Industry Applications, IEEE Transactions on 51 (2) (2015) $1249\{1258$. doi:10.1109/TIA.2014.2356639.
[11] P. Kanirajan, V. S. Kumar, Power quality disturbance detection and classification using wavelet and RBFNN, Applied Soft Computing 35(2015)470-481. doi:http://dx.doi.org/10.1016/j.asoc.2015.05.048.

[12] H. Eristi, O. Yildirim, B. Eristi, Y. Demir, Automatic recognition system of underlying causes of power quality disturbances based on s-transform and extreme learning machine, International Journal of Electrical Power and Energy Systems $61 \quad$ (2014) $553\{562$. doi:http://dx.doi.org/10. 1016/j.ijepes.2014.04.010.

[13] B. Panigrahi, P. Dash, and J. Reddy, "Hybrid signal processing and machine intelligence techniques for detection, quantification and classification of power quality disturbances," Engineering Applications of Artificial Intelligence, vol. 22, no. 3, pp. 442 - 454, 2009. [Online]. Available:http://www.sciencedirect.com/science/article/pii/ S0952197608001481

[14] A. K. Ghosh, D. L. Lubkeman, The classi_cation of power system disturbance waveforms using a neural network approach, Power Delivery, IEEETransactions on 10 (1) (1995) 109\{115. doi:10.1109/61.368408.

[15] M. Valtierra-Rodriguez, R. de Jesus Romero-Troncoso, R. A. Osornio- Rios, A. Garcia-Perez, Detection and classi_cation of single and combined power quality disturbances using neural networks, IEEE Transactions on Industrial Electronics $61 \quad$ (5) (2014) $2473\{2482$. doi:10.1109/TIE.2013. 2272276.

[16] Y.-L. Chen, H.-W. Hu, and K. Tang, "Constructing a decision tree from data with hierarchical class labels," Expert Systems with Applications, vol. 36, no. 3, Part 1, pp. 4838 - 4847, 2009. [Online]. Available: http://www.sciencedirect.com/science/article/pii/S0957417408002 73X.

[17] Y.-L. Chen, H.-W. Hu, K. Tang, Constructing a decision tree from data with hierarchical class labels, Expert Systems with Applications 36 (3, Part I 2009) 4838- 4847. doi:http://dx.doi.org/10.1016/j.eswa.2008.05.044.

[18] A. Rodriguez, J. A. Aguado, F. Martin, J. J. Lopez, F. Munoz, J. E. Ruiz,675 Rule-based classi_cation of power quality disturbances using s-transform, Electric Power Systems Research $86 \quad$ (2012) $113\{121$. doi:http://dx.doi.org/10.1016/j.epsr.2011.12.009.

[19] P. K. Ray, N. Kishor, S. R. Mohanty, Islanding and power quality disturbance detection in grid-connected hybrid power system using wavelet and s -transform, Smart Grid, IEEE Transactions on 3 (3) (2012) 1082\{1094. doi:10.1109/TSG.2012.2197642.

[20] S. Ventosa, C. Simon, M. Schimmel, J. J. Danobeitia, A. Manuel, The s-transform from a wavelet point of view, Signal Processing, IEEE Transactions on 56 (7) (2008) 2771 \{2780. doi:10.1109/TSP.2008.917029.

[21] S. Ventosa, C. Simon, M. Schimmel, J. J. Danobeitia, A. Manuel, The s-transform from a wavelet point of view, Signal Processing, IEEE Transactions on 56 (7) (2008) 2771 \{2780. doi:10.1109/TSP.2008.917029.

[22] R. H. G. Tan and V. K. Ramachandaramurthy, "Numerical model framework of power quality events," European Journal of Scientific Research, vol. 43, no. 1, pp. 30-47, June 2010.

\section{Creative Commons Attribution License 4.0 (Attribution 4.0 International, CC BY 4.0)}

This article is published under the terms of the Creative Commons Attribution License 4.0 https://creativecommons.org/licenses/by/4.0/deed.en_US 